\title{
Numerical Solution of Vibrating Double and Triple-Panel Stepped Thickness Plates
}

\author{
Mohamed A. El-Sayad* and Ahmed M. Farag
}

Department of Engineering Mathematics and Physics, Faculty of Engineering, Alexandria University, Alexandria, Egypt

\begin{abstract}
The main objective of the present paper is to achieve a modified numerical method for investigating the vibration characteristics of the stepped thickness plate with many types of boundary conditions surrounding certain number of panels. The presented technique relies on dividing the entire plate into several regions of uniform thickness separated by sudden steps. Each region is divided to number of strips which are assembled and solved numerically by the Finite Strip-Transition Matrix method FSTM. A convenient basic function is applied to reduce the partial differential equation of motion of plate inside a single region into an ordinary differential one. Step continuity conditions are applied to achieve the final solution of plate. Regional rigidities of plates and mass per unit area are changed due to the change of plate thickness from a region to another. Consequently, new straining actions are occurred and then compatibility conditions become necessary to modify the nodal vector at each step. Various types of restrained boundary conditions against rotation are included in the present paper. The validity of present method is checked and the accuracy of the results is compared with those available in literature showing a good agreement.
\end{abstract}

Keywords: Numerical; Transition; Stepped; Panel; Vibration; Plate

\section{Nomenclatures}

$W_{i}=W_{i}(z, h, t)$ Regional dimension-less plate displacement in the domain of region $R_{i} ; i=1,2,3$.

$\begin{array}{ll}a, b & \text { Plate dimension in } \zeta, \eta \text { directions respectively } \\ x, y & \text { Plate coordinates } \\ t & \text { Time } \\ \zeta, \eta & \text { Plate dimension-less coordinates; } \zeta=\frac{x}{a}, \eta=\frac{y}{b} \\ \beta=\frac{a}{b} & \text { Aspect ratio } \\ h_{i} & \text { Regional thickness of plate } \\ \rho & \text { Plate density } \\ M & \text { Number of panel } \\ k & \text { Parameter of homogenous sub-grade } \\ (\bar{m})_{i}=\frac{\rho}{g} h_{i} & \text { Plate mass per unit area } \\ (D)_{i}=\frac{E h_{i}^{3}}{\left(12-v^{2}\right)} & \text { Regional flexural rigidity of plate } \\ K_{G}=\frac{k a^{4}}{D_{1}} & \text { Normalized parameter of homogenous sub-grade } \\ k & \text { Parameter of homogenous sub-grade } \\ \omega & \text { Natural frequency } \\ \lambda_{m}^{2}=\omega^{2} a^{4}\left(\frac{m}{D}\right)_{1} & \text { Natural frequency parameter } \\ c_{m}, d_{m} & \text { Integral values } \\ \alpha_{i}=\frac{h_{1}}{h_{i}} & \text { Panel thickness ratio } \\ \left\{V_{m}\right\}_{i} & \text { Regional nodal vector } \\ {\left[Y_{K}\right]_{i}} & \text { Regional transition matrix } \\ \left\{V_{m}\right\}_{K_{j}},\left\{V_{m}\right\}_{K_{j-1}} & \text { Nodal vectors of strip } K_{j} \\ \Phi & \text { Restrained cofficient of rotation and translation at } \\ =0,1 & \end{array}$

$\varphi$

at $\eta=0,1$

$$
\begin{array}{ll}
\gamma_{i}=\left(\frac{h_{i}}{h_{i+1}}\right)^{3} ; i=1,2 & \text { Step thickness ratio } \\
v_{x} & \text { Poisson's ratio }
\end{array}
$$

\section{Introduction}

A certain structural optimization of a panel may be achieved by possessing a suitable variation of thickness of plate structure. The importance of types of structures increases when the aero-space and underground structures are considered. Survey of the literature on the flexural vibration of thin plate reveals that the work on this topic has been mainly confined to plates with uniform thicknesses. A relatively few studies have been published on the free vibration of isotropic plates of stepped thicknesses. During the past few decades, many researchers were devoted to mathematical modeling and numerical solution for static elastic multi-structure problems. Since vibration analysis of elastic structures plays important roles in engineering applications, this paper is concerned with Finite Strip-Transition Matrix method (FSTM) for vibration analysis of paneled stepped thickness plate.

Although, Chopra [1] has attempted an exact solution for a simply supported stepped thickness plate with two panels, Warburton [2] pointed out that continuity conditions used by Chopra [1] were incorrect. He presented a modified analytical technique for two paneled stepped plate with different properties of orthotropic. Sakata [3] proposed an approximate formula for estimating the fundamental

*Corresponding author: Mohamed A. El-Sayad, Department of Engineering Mathematics and Physics, Faculty of Engineering, Alexandria University, Alexandria, Egypt, E-mail: mel_sayad@hotmail.com

Received April 17, 2012; Accepted May 19, 2012; Published May 22, 2012

Citation: El-Sayad MA, Farag AM (2012) Numerical Solution of Vibrating Double and Triple-Panel Stepped Thickness Plates. J Appl Computat Math 1:110. doi:10.4172/2168-9679.1000110

Copyright: (c) 2012 El-Sayad MA, et al. This is an open-access article distributed under the terms of the Creative Commons Attribution License, which permits unrestricted use, distribution, and reproduction in any medium, provided the original author and source are credited. 
nature frequency of an isotropic plate with stepped thickness from the natural frequencies of the isotropic plate reduced from the orthotropic one. Recently, Farag [4] applied a closed form solution for vibrating surfaces of partially restrained and clamped double-panel plates via a power matrix exponential method. Xiang and Wang [5] also

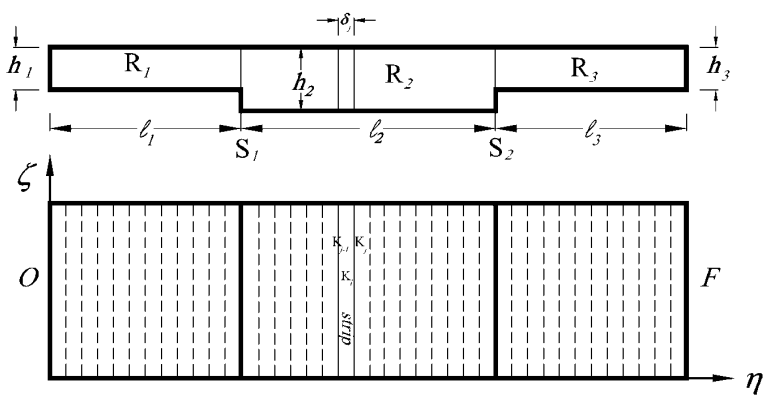

Figure 1: Finite Strips of Triple Panel Stepped Thickness plate.

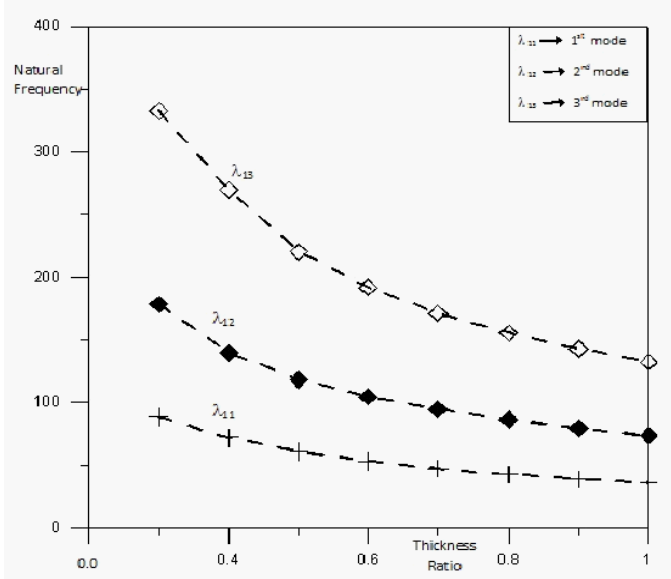

Figure 2-a: Relationships of the variation of natural frequency parameters $\lambda_{m n}$ due to the change of thickness ratio $\alpha_{2}$ for the first three modes where $m=1$ and $n=1,2$ or 3 for CCCC case.

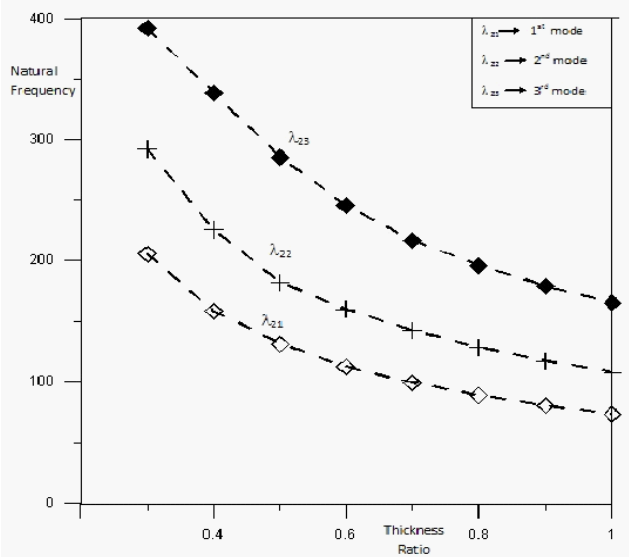

Figure 2-b: Relationships of the variation of natural frequency parameters $\lambda_{m n}$ due to the change of thickness ratio $\alpha_{2}$ or the first three modes where $m=2$ and $n=1,2$ or 3 for CCCC case.

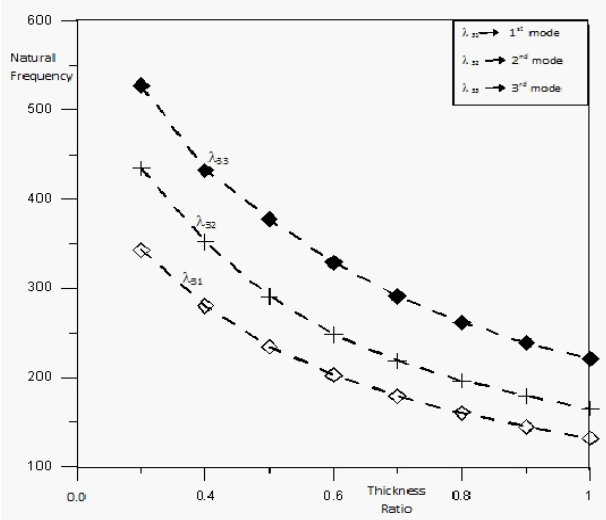

Figure 2-c: Relationships of the variation of natural frequency parameters $\lambda_{m n}$ due to the change of thickness ratio $\alpha_{2}$ for the first three modes where $m=3$ and $n=1,2$ or 3 for CCCC case.

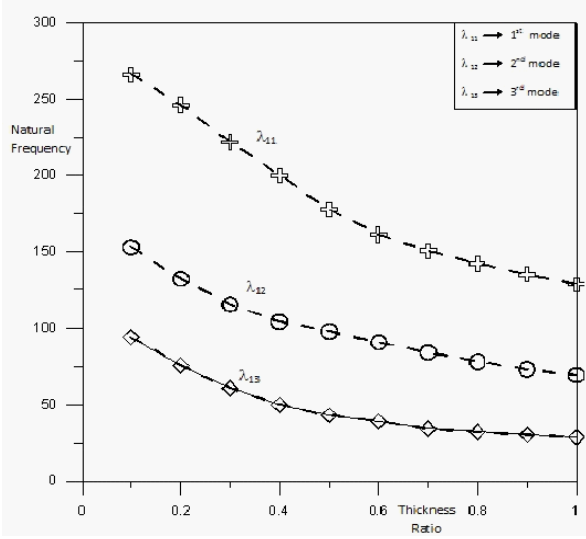

Figure 3-a: Relationships of the variation of natural frequency parameters $\lambda_{m n}$ due to the change of thickness ratio $\alpha_{2}$ for the first three modes where $m=1$ and $n=1,2$ or 3 for CSCS case.

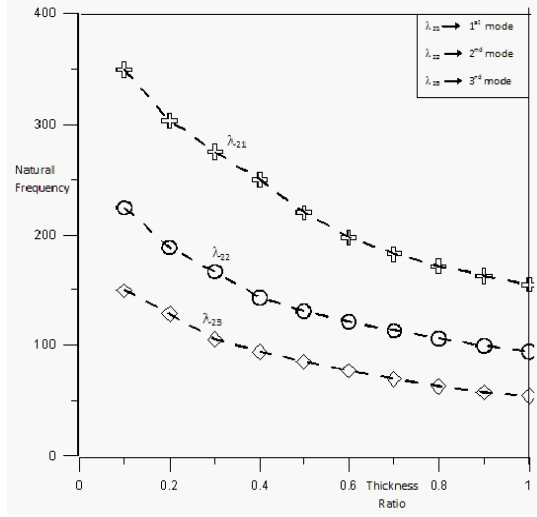

Figure 3-b: Relationships of the variation of natural frequency parameters $\lambda_{m n}$ due to the change of thickness ratio for the first three modes where $m=2$ and $n=1,2$ or 3 for CSCS case.

studied the exact vibration solutions of stepped rectangular plates. Xiang et al. [6,7] extended these studies in cooperation with others to include the case of stepped rectangular mindlin plate and the case of 
stepped circular mindlin plate. Gorman and Singha [8] outlined the vibration analysis of stepped cantilever plate using a supper position method. A numerical approach for rectangular stepped plate with sides restrained against rotation has been proposed by Laura and Filipich [9]. They ensured that vibration of plate with stepped thicknesses was studied in a very few number of literature. Filipich et al. [10] used a simple polynomial coordinate function which identically satisfied the restrained boundary conditions of plate with discontinuous thickness. Modal study via a discrete numerical approach, such as finite element method by Mukherjee and Mukhopadhyay [11], or finite strip method by Cheung and $\mathrm{Li}[12,13]$ introduces solution accuracy depending upon the suggested element size. Vibrations of plates with variable thickness were studied by Zanzi and Laura [14], Gutierrez et al. [15], Laura and Gutierrez [16] via different methods. Kaabi and Aksu $[17,18]$ applied a modified method to examine the dynamic behavior of rectangular plates with bilinear variation of thickness. Cheung and Li [19] achieved a simple finite strip method to analyze the hunched continuous bridges. Vibration of orthotropic rectangular plate with free edges in the case of discontinuously varying thickness was discussed by Laura et al. [20]. They extended their studies to include two other boundary conditions different from Laura et al. [21]. Farag [22] applied Finite Strip Transition Matrix method to solve the free and forced vibration problems of uniform thickness plate. In extended work, Farag [23] analyzed the stepped thickness plate analytically by means of the matrix exponential method. Recently, a semi and fully discrete finite element methods for investigating vibration analysis of elastic plate-plate structures are proposed by Junjiam et al. [24]. Semie [25] explained the numerical modeling of thin plates using the finite element method. Sanches et al. [26] studied the dynamic stationary response of reinforced plate by the boundary element method.

The present paper offers a modified numerical method relying on reducing the partial differential equation of motion into an ordinary differential one. A convenient basic function is used to obtain the later equation which is solved by Finite Strip Transition Matrix Method (FSTM) inside each panel of plate. Due to the sudden change of thickness between two adjacent panels, continuity conditions must be satisfied to generate the final solution of the entire plate. The known basic function is expressed in a variable parallel to the steps. The reduced differential equation of rectangular stepped plate is still carrying the other unknown variable in the other plate direction. The restrained boundary conditions are included and employed to derive the solution of the particular cases of clamped and simply supported edges.

\section{Equation of Motion}

The isotropic rectangular plates with elastically restrained boundary conditions in the two directions $\zeta$ and $\eta$ of plates are considered here. The plate thickness shown in figure 1 is a uniform in $\zeta$-direction, while it changes suddenly in $\eta$-direction at a step $S_{i}$ from $h_{i}$ to $h_{i+1}$.

Referring to the domain of the isotropic region $R_{i}$, the regional dimensionless partial differential equation of motion of plate vibration is:

$$
\bar{\nabla}^{4} W_{i}+\left(\frac{\bar{m} a^{4}}{D}\right)_{i} \frac{\partial^{2} W_{i}}{\partial t^{2}}+\left(\frac{k a^{4}}{D}\right)_{i} W_{i}=0 ; \quad i=1,2,3
$$

Where

$$
\bar{\nabla}^{2}=\frac{\partial^{2}}{\partial x^{2}}+\beta^{2} \frac{\partial^{2}}{\partial y^{2}}
$$

The different symbols are denoted in the nomenclatures part where the suffix $i$ means that the mentioned magnitudes are calculated inside the region $R_{i}$.

\section{Method of Solution}

The partial differential equation (1) is solved numerically under the proposed boundary conditions at $(\zeta=0,1)$ and $(\eta=0,1)$ by using the Technique of Finite Strip-Transition Matrix FSTM. In this Technique, the investigated plate is divided into three stepped panels which are separated by a number M-1 of steps. Each individual panel is divided

\begin{tabular}{|c|c|c|c|c|c|c|c|c|}
\hline \multirow{2}{*}{$\alpha_{2}=h_{1} / h_{2}$} & \multirow{2}{*}{$\sigma_{m n}=\frac{\lambda_{m n}}{\pi^{2}}$} & \multicolumn{3}{|c|}{ SSSS; } & \multicolumn{3}{|c|}{ CSCS; } & \multirow{2}{*}{$\begin{array}{l}\text { CCCC; } \\
\text { Present }\end{array}$} \\
\hline & & Present & Xiang et al. [5] & Farag [4] & Present & Xiang et al. [5] & Farag [4] & \\
\hline & $\sigma_{11}$ & 02.9015 & 02.9015 & 02.9015 & 04.1711 & 04.1711 & 04.1711 & 05.3028 \\
\hline \multirow[t]{3}{*}{$1 / 2$} & $\sigma_{12}$ & 07.1157 & 07.1156 & 07.1156 & 09.9048 & 09.9047 & 09.9047 & 10.5159 \\
\hline & $\sigma_{13}$ & 13.7849 & 13.7850 & 13. 7848 & 18. 0455 & 18.0450 & 18. 0453 & 18.6238 \\
\hline & $\sigma_{11}$ & 02.4470 & 2.4471 & 02.4470 & 03.5609 & 03.5610 & 03.5609 & 04.4631 \\
\hline \multirow[t]{2}{*}{$2 / 3$} & $\sigma_{12}$ & 06.2230 & 6.2229 & 06.2229 & 08.7200 & 08.7199 & 08.7199 & 09.2528 \\
\hline & $\sigma_{13}$ & 11.9125 & 11.9480 & 11.9124 & 15.6217 & 15.6210 & 15.6215 & 16.0596 \\
\hline
\end{tabular}
to a number $\mathrm{N}$ of finite strips separated by a number $\mathrm{N}+1$ of nodal

\begin{tabular}{|c|c|c|c|c|c|c|}
\hline \multirow[b]{2}{*}{$\alpha_{2}, \alpha_{3}$} & \multicolumn{6}{|c|}{ Natural frequencey parameters $\lambda_{m n}$} \\
\hline & $\lambda_{11}$ & $\lambda_{12}$ & $\lambda_{13}$ & $\lambda_{14}$ & $\lambda_{15}$ & $\lambda_{16}$ \\
\hline $1.0,1.0$ & 35.9195 & 73.5929 & 132.3785 & 211.2511 & 309.0456 & 416.1822 \\
\hline $0.9,1.0$ & 37.9563 & 77.3438 & 138.6326 & 222.3554 & 327.3623 & 437.8916 \\
\hline $0.8,1.0$ & 40.5830 & 81.3422 & 145.6952 & 236.6180 & 348.0211 & 459.2436 \\
\hline $0.7,1.0$ & 43.9586 & 85.5344 & 154.3948 & 236.6180 & 370.3955 & 480.2570 \\
\hline $0.6,1.0$ & 46.3017 & 71.6753 & 102.2939 & 255.4175 & 332.9891 & 385.8950 \\
\hline $0.5,1.0$ & 54.0737 & 95.5105 & 184.6845 & 267.9179 & 410.2973 & 539.8316 \\
\hline $0.4,1.0$ & 62.4249 & 104.1121 & 215.7646 & 343.2175 & 425.5430 & 616.2196 \\
\hline $0.3,1.0$ & 76.9445 & 122.0380 & 268.1892 & 366.2887 & 447.2614 & 819.1406 \\
\hline $0.2,1.0$ & 109.3091 & 168.1862 & 333.4251 & 376.4200 & 543.4720 & 1265.0045 \\
\hline $0.1,1.0$ & 215.0652 & 311.9320 & 359.3840 & 410.0752 & 1080.9598 & 2590.7068 \\
\hline
\end{tabular}

Table 1: Comparesons of the natural frequency coefficient $\sigma_{m n}$ for square Plates SSSS and CSCS.

Table 2: The natural frequency parameter $\lambda_{m n}$ for 3-panels square clamped plate CCCC. 
Citation: El-Sayad MA, Farag AM (2012) Numerical Solution of Vibrating Double and Triple-Panel Stepped Thickness Plates. J Appl Computat Math 1:110. doi:10.4172/2168-9679.1000110

Page 4 of 7

lines as in figure 1. The basic function of strip [23], is applied to reduce the partial differential equation of motion into an ordinary differential one. The reduced differential equation is solved numerically by the FSTM as an initial value problem under the proposed initial boundary conditions at $(\eta=0)$. The initial nodal conditions of each strip are applied to derive the strip end nodal conditions which are used as initial nodal conditions to the next strip. All nodal straining actions of each strip are obtained until the end of the current panel is reached. Because the intermediate end of a current panel is sudden step, the initial nodal conditions of the next panel must be modified by satisfying the compatibility conditions at this step. The FSTM is applied for all strips of all panels until the final boundary end of plate is reached. Satisfying the boundary conditions at $(\eta=1)$, one can obtain the final solution of the equation motion of plate.

\section{Reduction technique}

The regional displacement of the stepped plate is:

$$
W_{i}(\zeta, \eta, t)=\sum_{m=1}^{\mathrm{M}}\left(U_{m}(\zeta)\right)_{i}\left(V_{m}(\eta)\right)_{i} \sin \omega t
$$

Where $\left(U_{m}(\zeta)\right)_{i}$ is known regional shape function satisfying the plate boundary conditions [23] at $(\zeta=0,1)$ and $\left(V_{m}(\eta)\right)_{i}$ is unknown regional longitudinal function to be determined at $(\eta=0,1)$.

Eq. (2) is applied to reduce the partial differentia Eq. (1) Inside the Region $R_{i} ; i=1,2,3$ into [4]:

$$
\left(V_{m}^{\prime \prime \prime \prime}\right)_{i}+\frac{2 c_{m}}{\beta^{2}}\left(V_{m}^{\prime \prime}\right)_{i}+\frac{1}{\beta^{4}}\left[K_{G} D_{1}\left(\frac{1}{D}\right)_{i}-\lambda_{m}^{2} \frac{D_{1}}{h_{1}}\left(\frac{h}{D}\right)_{i}+d_{m}\right]\left(V_{m}\right)_{i}=0
$$

Where

$$
\begin{array}{r}
K_{G}=\frac{k a^{4}}{D_{1}}, \lambda_{m}^{2}=\omega^{2} a^{4}\left(\frac{\bar{m}}{D}\right)_{1}, c_{m}=\frac{\int_{0}^{1} U_{m} U_{m}^{\prime \prime} d \zeta}{\int_{0}^{1} U_{m} U_{m} d \zeta}, c_{m}=\frac{\int_{0}^{1} U_{m} U_{m}^{\prime \prime} d \zeta}{\int_{0}^{1} U_{m} U_{m} d \zeta}, \\
d_{m}=\frac{\int_{0}^{1} U_{m} U_{m}^{\prime \prime \prime} d \zeta}{\int_{0}^{1} U_{m} U_{m} d \zeta}
\end{array}
$$

\begin{tabular}{|c|c|c|c|c|c|c|c|}
\hline \multirow[b]{2}{*}{$\alpha_{2}, \alpha_{3}$} & \multirow[b]{2}{*}{$\lambda_{m n}$} & \multicolumn{6}{|c|}{ Restraint coefficients against rotation $\varphi_{o}=\varphi_{F}$} \\
\hline & & 0.0 & 0.02 & 0.2 & 2.0 & 200 & $\infty$ \\
\hline \multirow[t]{3}{*}{$1.0,1.0$} & $\lambda_{11}$ & 35.9195 & 34.7141 & 31.1493 & 29.1644 & 28.8413 & 28.8379 \\
\hline & $\lambda_{12}$ & 73.5929 & 69.7890 & 50.1225 & 55.6106 & 54.9253 & 54.9181 \\
\hline & $\lambda_{13}$ & 132.3787 & 125.0569 & 109.4910 & 103.5695 & 102.7365 & 102.7279 \\
\hline \multirow[t]{3}{*}{$0.75,1.0$} & $\lambda_{11}$ & 42.1654 & 40.8311 & 37.1138 & 35.1818 & 34.8760 & 34.7828 \\
\hline & $\lambda_{12}$ & 83.4136 & 78.8536 & 67.9529 & 63.1631 & 62.4507 & 62.4432 \\
\hline & $\lambda_{13}$ & 149.7636 & 141.3653 & 124.3771 & 118.1902 & 117.3307 & 117.3219 \\
\hline \multirow[t]{3}{*}{$0.5,1.0$} & $\lambda_{11}$ & 54.0736 & 52.5033 & 48.5825 & 46.7700 & 46.4956 & 46.4927 \\
\hline & $\lambda_{12}$ & 95.5105 & 90.5687 & 79.5519 & 57.0258 & 74.3679 & 74.3610 \\
\hline & $\lambda_{13}$ & 184.8631 & 175.7379 & 157.7919 & 151.4550 & 150.5831 & 150.7542 \\
\hline \multirow[t]{3}{*}{$0.25,1.0$} & $\lambda_{11}$ & 84.6291 & 88.3274 & 58.8198 & 84.7820 & 84.6307 & 84.6291 \\
\hline & $\lambda_{12}$ & 123.5494 & 135.5536 & 127.3214 & 124.0274 & 123.5543 & 123.5494 \\
\hline & $\lambda_{13}$ & 233.6159 & 282.3131 & 246.3862 & 235.1273 & 233.6312 & 233.6164 \\
\hline
\end{tabular}

Consequently, the equation of motion for any panel becomes:

$$
\left(V_{m}^{\prime \prime \prime \prime}\right)_{i}=\left[\begin{array}{llll}
\frac{-1}{\beta^{4}}\left(\alpha_{i}^{3} K_{G}-\alpha_{i}^{2} \lambda_{m}^{2}+d_{m}\right) & 0 & \left(-2 \frac{c_{m}}{\beta^{2}}\right) & 0
\end{array}\right]_{i}\left\{\begin{array}{c}
V_{m} \\
V_{m}^{\prime} \\
V_{m}^{\prime \prime} \\
V_{m}^{\prime \prime \prime}
\end{array}\right\}_{i}
$$

Table 3: The natural frequency parameter $\lambda_{m n}$ for 3-panels square clamped restrained plate $C E_{R} C E_{R}$.

\begin{tabular}{|c|c|c|c|c|c|c|}
\hline & & \multicolumn{5}{|c|}{ Restraint coefficients against rotation $\varphi_{o}=\varphi_{F}$} \\
\hline$\alpha_{2}, \alpha_{3}$ & $\lambda_{m n}$ & 0.0 & 0.1 & 1.0 & 100 & $\infty$ \\
\hline $0.75,1.0$ & $\lambda_{11}$ & 26.7406 & 24.9157 & 23.1746 & 22.7619 & 22.7573 \\
\hline & $\lambda_{12}$ & 66.0149 & 60.3497 & 56.4543 & 55.6976 & 55.6894 \\
\hline & $\lambda_{13}$ & 128.6246 & 118.5003 & 113.3414 & 112.4694 & 112.4602 \\
\hline $0.5,1.0$ & $\lambda_{11}$ & 31.4907 & 29.3873 & 27.6339 & 27.2489 & 27.2447 \\
\hline & $\lambda_{12}$ & 75.0484 & 68.5108 & 64.6539 & 63.9483 & 63.9407 \\
\hline & $\lambda_{13}$ & 160.2106 & 149.9871 & 144.8592 & 143.9884 & 143.9792 \\
\hline $0.75,1.0$ & $\lambda_{11}$ & 47.4033 & 43.0552 & 38.3594 & 37.1537 & 37.1398 \\
\hline & $\lambda_{12}$ & 135.0159 & 121.8370 & 112.2586 & 110.3528 & 110.3321 \\
\hline $0.5,1.0$ & $\lambda_{13}$ & 275.0068 & 251.6332 & 239.4639 & 237.3944 & 237.3724 \\
\hline & $\lambda_{11}$ & 53.3631 & 48.4637 & 43.7366 & 42.6063 & 42.5936 \\
\hline & $\lambda_{12}$ & 147.6858 & 132.0205 & 122.2170 & 120.3904 & 120.3904 \\
\hline & $\lambda_{13}$ & 338.6052 & 315.2836 & 303.4671 & 301.4525 & 301.4311 \\
\hline & $\lambda_{11}$ & 77.0914 & 69.2508 & 60.3105 & 57.9252 & 57.8977 \\
\hline & $\lambda_{12}$ & 231.7011 & 207.9731 & 190.3487 & 186.8081 & 186.7695 \\
\hline & $\lambda_{13}$ & 479.9336 & 437.9678 & 415.9456 & 412.1925 & 412.1526 \\
\hline & $\lambda_{11}$ & 85.5963 & 76.9994 & 68.1073 & 65.8863 & 65.8610 \\
\hline & $\lambda_{12}$ & 249.6733 & 220.5666 & 202.1801 & 198.7270 & 198.7270 \\
\hline & $\lambda_{13}$ & 588.0605 & 546.3231 & 525.1012 & 521.4784 & 521.4784 \\
\hline
\end{tabular}

Table 4: The natural frequency parameter $\lambda_{m n}$ for 3-panels square simply supported- restrained plate $\mathrm{SE}_{\mathrm{R}} \mathrm{SE}_{\mathrm{R}}$. 


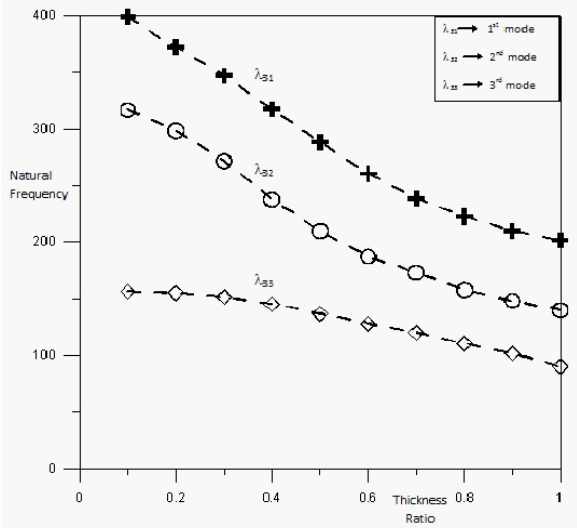

Figure 3-c: Relationships of the variation of natural frequency parameters $\lambda_{m n}$ due to the change of thickness ratio $\alpha_{2}$ for the first three modes where $m=3$ and $n=1,2$ or 3 for CSCS case.

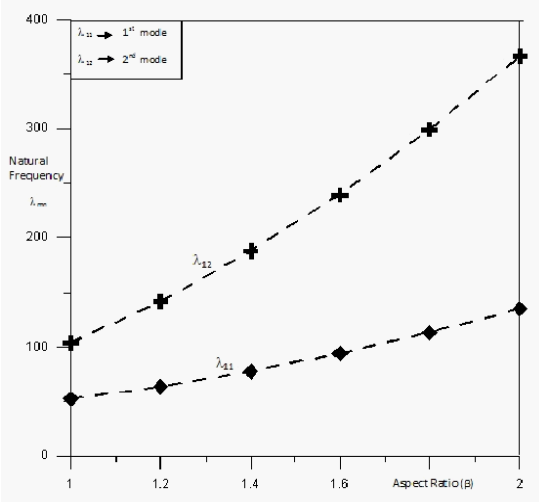

Figure 4-a: Relationship of the variation of natural frequency parameters $\lambda_{m n}$ due to the change of aspect ratio $\beta$ for the first two modes where $m=1$ and $n=1,2$ or 3 for CCCC case

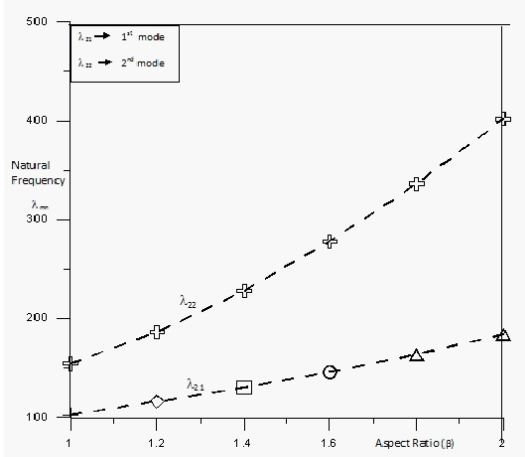

Figure 4-b: Relationships of the variation of natural frequency parameters $\lambda_{m n}$ due to the change of aspect ratio $\beta$ for the first two modes where $m=2$ and $n=1,2$ or 3 for CCCC case.

where:

$\alpha_{i}=\frac{h_{1}}{h_{i}}$ is the panel thickness ratio at region $R_{i} ; i=1,2,3$

The ordinary differential equation (4) is transformed within a region $R_{i}$ to a linear system of differential equations expressed as [22]:

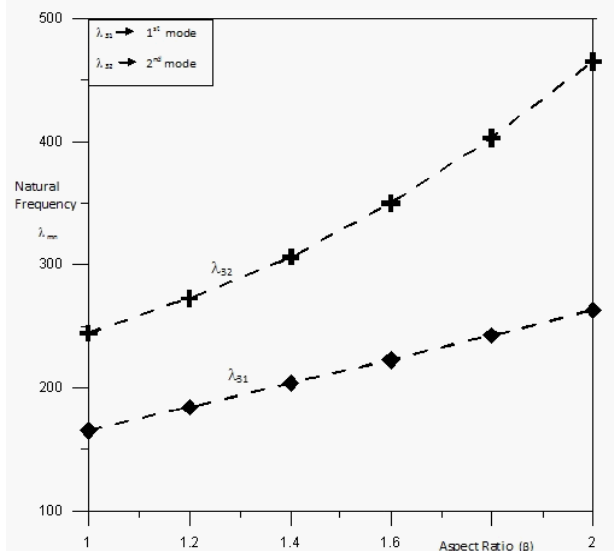

Figure 4-c: Relationships of the variation of natural frequency parameters $\lambda_{m n}$ due to the change of aspect ratio $\beta$ for the first two modes where $m=3$ and $n=1,2$ or 3 for CCCC case.

$\frac{d}{d \eta}\left\{V_{m}\right\}_{i}=\left[A_{m}\right]_{i}\left\{V_{m}\right\}_{i}$

Where

$\left\{V_{m}\right\}_{i}=\left\{\begin{array}{c}V_{m} \\ V_{m}^{\prime} \\ V_{m}^{\prime \prime} \\ V_{m}^{\prime \prime \prime}\end{array}\right\}_{i}$

And

$\left[A_{m}\right]_{i}=\left[\begin{array}{cccc}0 & 1 & 0 & 0 \\ 0 & 0 & 1 & 0 \\ 0 & 0 & 0 & 1 \\ \frac{-1}{\beta^{4}}\left(\alpha_{i}^{3} K_{G}-\alpha_{i}^{2} \lambda_{m}^{2}+d_{m}\right) & 0 & \left(-2 \frac{c_{m}}{\beta^{2}}\right) & 0\end{array}\right]$

The general solution of the ordinary differential equation is:

$$
\left\{V_{m}\right\}_{K_{j}}=\left[Y_{K}\right]_{i}\left\{V_{m}\right\}_{K_{j-1}}
$$

Where $\delta_{i}$ is the width of strip $\kappa_{j}$ bounded by two nodal lines $K_{j}, K_{j-1}$ inside region $R_{i}$.

The regional Transition Matrix $\left[Y_{K}\right]_{i}$ is calculated according to the Runge Kutta method such as:

$$
\left[Y_{K}\right]_{i}=I+\sum_{r=1}^{4} \frac{\left[A_{m}\right]_{i}^{r}\left(\delta_{i}\right)^{r}}{r !}
$$

Where $I$ is the unit matrix?

\section{Continuity conditions}

The Magnitudes of displacement $W$ slope $\frac{\partial W}{\partial v}$ Moment $M_{V}$ and shear $Q_{v}$ must satisfy the continuity conditions at the sudden step $S$ so that: 


$$
\left\{\begin{array}{c}
V_{m} \\
V_{m}^{\prime} \\
V_{m}^{\prime \prime} \\
V_{m}^{\prime \prime \prime}
\end{array}\right\}_{\mathfrak{R} i}=\left\{\begin{array}{c}
V_{m} \\
V_{m}^{\prime} \\
v_{x} c_{m}\left(\gamma_{i}-1\right) \beta^{-2} V_{m}+\gamma_{i} V^{\prime \prime} \\
c_{m}\left(2-v_{x}\right)\left(\gamma_{i}-1\right) \beta^{-2} V_{m}^{\prime}+\gamma_{i} V^{\prime \prime \prime}
\end{array}\right\}_{L i}
$$

The symbols $\mathfrak{R}_{i}, L_{i}$ denote positions immediately after and before the sudden step $S_{i}$ respectively. Where $\gamma_{i}=\left(\frac{h_{i}}{h_{i+1}}\right)^{3}$ is step thickness ratio at step $S_{i} ; i=1,2$ and $v_{x}$
is Poisson's ratio.

A right step vector $\left\{V_{m}\right\}_{\mathfrak{\Re}_{i}}$ of the sudden step $S_{i}$ is achieved by updating the step left vector $\left\{V_{m}\right\}_{L_{i}}$ due to Eq. (10).

\section{Boundary and initial conditions}

Applying the proposed boundary conditions at $\eta=0$, one can reduce the four initial unknowns $V_{m}(0), V_{m}^{\prime}(0), V_{m}^{\prime \prime}(0)$ and $V_{m}^{\prime \prime \prime}(0)$ to only two unknowns. Consequently, by satisfying the boundary conditions of plate at $\eta=1$, two characteristic equations for the plate vibration are established. The natural frequency parameters of plate are the Eigen values of the characteristic matrix of these equations. The corresponding Eigen vectors create the mode shapes. Boundary conditions at the edges $\eta=0,1$ are considered for various types of edges [22], such as simply supported S, clamped C, and free F, elastically restrained against rotation $E_{R}$. The restrained coefficients of rotation $\Phi \eta=0,1$ are usually applied to vary from 0 to $\infty$.

The initial conditions are expressed as initial values $V_{m}(0), V_{m}^{\prime}(0), V_{m}^{\prime \prime}(0)$ and $V_{m}^{\prime \prime \prime}(0)$ which are the components of initial vector $\left\{V_{m}\right\}_{o}$ of the first region $R_{1}$. This initial vector $\left\{V_{m}\right\}_{o}$ can be derived according to the known boundary conditions at the first nodal edge $\eta=0$ [23].

\section{Results and Discussions}

Different cases of plates composed of different panels with unequal thicknesses and panel widths are investigated by the present technique. This study takes in account the variation of the aspect ratios $\beta$ and various magnitudes of panel thickness ratio $\alpha_{i} ; i=1,2,3$ and the coefficients of partially restrained boundary conditions. The natural frequency parameter $\lambda_{m n}$ is calculated for every case where Poisson's ratio is taken as $v=0.3$.

The natural frequencey coefficients $\sigma_{m n}=\frac{\lambda_{m n}}{\pi^{2}}$ based on the present technique are compared as shown in Table 1 with those obtained by Xiang et al. [5] using an exact solution and using closed form solution [4].

The comparisons are available only for the case of double panel square plate with panel width ratio $\ell_{1}:\left(\ell_{1}+\ell_{2}\right)=1: 2$ as shown in Table 1. Calculations are carried out in two cases of boundary conditions of square plates SSSS and CSCS where S, C mention to the simply supported and clamped edges respectively. The general method of restrained boundary conditions is applied when $\Phi_{1}=\Phi_{2}=\infty$ , $\varphi_{o}=\varphi_{F}=\infty$ to posses the case of full simply supported plate SSSS. Another case of plate CSCS is obtained where $\Phi_{1}=\Phi_{2}=\infty$ and $\varphi_{o}=\varphi_{F}=0$. The third case is full clamped plate CCCC where $\Phi_{1}=\Phi_{2}=0$ and $\varphi_{o}=\varphi_{F}=0$. The results are obtained for panel thickness ratios equal to 0.5 and $2 / 3$. The comparisons show excellent agreement.
The natural frequencey parameters $\lambda_{m n}$ in the first six modes are obtained in Table 2 for the cases of 3-panels square full clamped plates CCCC with panel width ratio $\ell_{1}: \ell_{2}: \ell_{3}=0.25: 0.50: 0.25$. The results are calculated under the variation of $\alpha_{2}$ from 1.0 to 0.1 where $\alpha_{1}=\alpha_{3}=1$. The results show that the natural frequency parametrer inreases by decreasing $\alpha_{2}$.

Table 3 shows the natural frequencey parameters $\lambda_{m n}$ for the cases of 3- panels square full plates $\mathrm{CE}_{R} \mathrm{CE}_{\mathrm{R}}$ with two opposite edges clamped and other edges ellastically restrained against rotation with restrained coefficient varying from 0 to $\infty$. The results are obtained for cases of panel width ratio $\ell_{1}: \ell_{2}: \ell_{3}=0.25: 0.50: 0.25$ and $\alpha_{2}$ varying from 1.0 to 0.25 , where $\alpha_{1}=\alpha_{3}=1$. The natural frequencey parameter is obtained for the first three modes. The results show that the natural frequency parametrer decreases by increasing the coefficient of restrained $\varphi$.

This case is carried out for 3-panel square plate, $\mathrm{SE}_{\mathrm{R}} \mathrm{SE}_{\mathrm{R}}$, simply supported at two opposite edges and partially restrained against rotation at the other edges (Table 4 ). The results are obtained for panel width ratios $\ell_{1}: \ell_{2}: \ell_{3}=0.25: 0.50: 0.25$ and panel thickness ratio $1: \alpha_{2}: 1$ where $\alpha_{2}$ varies from 1.0 to 0.25 . Table 4 shows that the natural frequency parameter increases by decreasing the restrained coefficient $\varphi$. For the double panel isotropic, square, clamped supported plate at all edges CCCC the relationships of the variation of natural frequency parameters due to the change of thickness ratio $\alpha_{2}$ are plotted in figures 2 -a,b,c. For the panel width ratio $\ell_{1}:\left(\ell_{1}+\ell_{2}\right)=1: 3$, the natural frequency parameters $\lambda_{m n}$ are obtained for the first three modes where $m=1,2$ or 3 and $n=1,2$ or 3 .

Similarly, the case of square isotropic stepped plate CSCS with two opposite edges clamped and other edges simply supported is carried out as shown in figures 3-a,b,c. The frequency parameters $\lambda_{m n}$ are recorded for the case of panel width ratio $\ell_{1}:\left(\ell_{1}+\ell_{2}\right)=1: 2$ when the thickness ratio $\alpha_{2}$ changes from 0.1 to 1.0 . The results show that the natural frequency parameters increase by decreasing the thickness ratio $\alpha_{2}$ for all modes.

The case of full clamped stepped rectangular plate CCCC with thickness ratio $\alpha_{2}=.5$, panel width ratio $\ell_{1}:\left(\ell_{1}+\ell_{2}\right)=1: 2$ and aspect ratio $\beta$ of the intire plate varying from 1 to 2 is investigated as shown in figures $4-\mathrm{a}, \mathrm{b}, \mathrm{c}$. The resuls show that the parameter $\lambda_{m n}$ increases by increasing the aspect ratio $\beta$ for all recorded modes.

\section{Conclusion}

The finite strip transition matrix method FSTM described here involvoes a numerical solution of stepped paneled plate with classical and restrained boundary conditions. This method is a combination between the strip and transition matrix method to solve the vibration problem of stepped plates as an initial value proplem. Transition matrix method is a semi analytical method relying on estmiating the numerical solution of the intial value problem by means of Range Kutta method. The plate domain is divided into paneled regoins consisting of strips bounded by nodal lines. Each strip is governed by the transition matrix formula which transite from one strip to another via nodal vectors until the final edge is reached. Several cases of double and triple panel plates are investigated for the variation of thickness ratio, aspect ratio, panel width ratio and boundary conditions. To show the accuracy of the present method, the results have been summerized and compared with those obtained by other methods. A good exteremely agreement of results is found for all compared cases. 
Citation: El-Sayad MA, Farag AM (2012) Numerical Solution of Vibrating Double and Triple-Panel Stepped Thickness Plates. J Appl Computat Math 1:110. doi:10.4172/2168-9679.1000110

\section{References}

1. Chopra (1974) Vibration of Stepped Thickness Plates. Int J Sci 16: 337-344.

2. Warburton $\mathrm{G}(1975)$ Letter to the Editor Comment on: Vibration of Stepped Thickness Plates by Chopra, Int J Sci 17: 239.

3. Sakata T (1981) Natural Frequencies of Orthotropic Rectangular Plates with Stepped Thickness, J Sound Vib 74: 73-79.

4. Farag A (2009) Closed Form Solution for Vibrating Surfaces of Partially Restrained and Clamped Double-Panel Plates. European Journal of Scientific Research 29: 320-333.

5. Xiang Y, Wang CM (2002) Exact Buckling and Vibration Solution for Stepped Rectangular Plates. J Sound Vib 250: 503-517.

6. Xiang Y, Wei GW (2004) Exact Solution for Buckling and Vibration of Stepped Rectangular Mindlin Plates. Int J Solids Struct 41: 279-294.

7. Xiang Y, Zhang L (2005) Free Vibration Analysis of Stepped Circular Mindlin Plates. J Sound Vib 280: 633-655

8. Gorman DJ, Singhal R (2002) Free Vibration analysis of Cantilever Plates with Steps Discontinuities in Properties by the Method of Superposition, J Sound Vib 253: 631-652.

9. Laura PAA, Filipich C (1977) Fundamental Frequency of Vibration of Stepped Thickness Plates. J Sound Vib 50: 157-158.

10. Filipich C, Laura PAA, Gianetti CE, Luisoni LE (1978) Vibrations of Rectangular, Stepped Thickness Plates with Edges Elastically Restrained Against Rotation, Int J Mech Sci 20: 149-158.

11. Mukherjee A, Mukhopadhyay M (1988) Finite Element Vibration of Eccentrically Stiffened Plates. Comput Struct 30: 1303-1317.

12. Cheung YK, Kong J (1995) Application of a new finite strip to Free Vibration of Rectangular Plates of Varying Complexity, J Sound Vib 181: 341-353.

13. Cheung MS, Li W (1990) Analysis Of Haunched, Continuous Bridge by Spline Finite Strips. Comput Struct 36: 297-300.

14. Laura PAA, Valerga de Greco BH (1988) Numerical experiments on the determination of the fundamental frequency of transverse vibration of nonuniform rectangular plates. J Sound Vib 123: 382-386.
15. Gutierrez RH, Rossi RE, Laura PAA (1995) Determination of the Fundamental Frequency of Transverse Vibration of Rectangular Plates when the Thickness Varies in A Discontinuous Fashion. Ocean Engineering 22: 663-668.

16. Laura PAA, Gutierrez RH (2002) Transverse Vibration of Rectangular Plates of Generalized Anisotropy and Discontinuously varying thickness. J Sound Vib 250: 569-574.

17. AL-Kaabi SA, Aksu G (1988) Natural Frequencies of Mindlin Plates of Bilinearly Varying Thickness, J Sound Vib 123: 373-379.

18. Aksu G, Al- Kaabi SA (1987) Free Vibration Analysis of Mindlin Plates of Linearly Varying Thickness, J Sound Vib 119: 189-205.

19. Cheung MS, Li W (1990) Analysis Of Haunched Continuous Bridge by Spline Finite Stresses, Comput Struct 36: 297-300.

20. Laura PAA, Bambill DV, Rossi RE, Rossit CA (1997) Vibration of Orthotropic Rectangular Plate with a free Edge in the Case of Discontinuously Varying Thickness. J Sound Vib 206: 109-113.

21. Bambill DV, Rossi RE, Laura PAA, Rossit CA (1998) Vibration of Orthotropic Rectangular Plate with of Non Uniform and Two Adjacent Free Edges. J Sound Vib 215: 189-194.

22. Farag A (1994) Mathematical Analysis of Free and Forced Vibration of Rectangular Plate. Ph.D. Thesis, Faculty of Engineering, Alexandria University Alexandria.

23. Farag A (2007) Analytical Method for Vibration Analysis of Stepped Thickness Plates. Proceedings of the 8th International Conference on Concrete Technology in Developing Countries Hammamat-Tunisia, 8-9 November 2007.

24. Jumjiang L, Jianguo H, Zhimin Z (2009) A Finite Method for Vibration Analysis of Elastic Plate-Plate Structures. Journal of Discrete and Continuous Dynamical Systems Series B.

25. Semie A (2010) Numerical Modelling of Thin Plates using the Finite Element Method. M.Sc. Thesis, Department of Computational Science, Addis Ababa University.

26. Sanches LCF, Mesquita E, Pavanello R, Palermo L Jr (2007) Dynamic Stationary Response of Reinforced Plates by the Boundary Element Method. Hindawi Publishing Corporation Mathematical Problems in Engineering. 